\title{
IMAPlate Based Miniature, High Sensitive, Rapid Screening Method for Detecting Bioengineered, Secreted Lipase Activities in Yeast Expression Systems
}

\author{
Michel A. Sciottia , Guo Jun Chen ${ }^{\mathrm{b}}$, and Daniel Gygax ${ }^{\star a}$
}

\begin{abstract}
A spectrophotometric assay based on a miniaturized 96-well plate device (IMAPlate) enables a rapid and simple screening of bioengineered recombinant lipases expressed and secreted by Saccharomyces cerevisiae. Starting from a colony, the test delivers a quantitative estimation of enzymatic activity titer in $24 \mathrm{~h}$ or less with manual high throughput performances.
\end{abstract}

Keywords: Enzymatic assay · IMAPlate · Miniaturized reactor · Protein expression · Screen

\section{Introduction}

Lipases (EC 3.1.1.3) catalyze a wide range of cofactor-independent reactions, typically hydrolysis of esters in aqueous solution, but also esterifications and transesterifications in solvents. Industrial applications involving lipases in detergent powders as a fat degradation agent or exploiting their stereo-specific property for the production of optical pure compound are already widespread..1] Recent works set a focus on developing processes for lipase-driven production of polymers, fine chemicals or biodiesel.[2] Therefore, extensive protein engineering strategies were undertaken to stress the natural properties of these enzymes in terms of kinetics, heat resistance and solvent tolerance. ${ }^{[3]}$

Candida Antarctica lipase B (CALB, EC 3.1.1.3) demonstrates good hydrolysis and esterification abilities. In the past decade, it has been studied by random mutagenesis approaches and structure model-based protein design. ${ }^{[4-6]}$ CALB is successfully expressed and secreted as a

\footnotetext{
${ }^{*}$ Correspondence: Prof. Dr. D. Gygaxa

Tel.: +41614674562

Fax: +41614674701

E-mail: daniel.gygax@fhnw.ch

aHochschule für Life Sciences

Fachhochschule Nordwestschweiz

Gründenstrasse 40

$\mathrm{CH}-4132$ Muttenz

bNCL New Concept Lab

Eichenstrasse 22

$\mathrm{CH}-4313$ Möhlin
}

recombinant protein in a variety of fungal expression systems, mainly yeasts..$^{[7,8]}$ But these organisms generally require long incubation time for growth and protein synthesis, consequently lowering the throughput of screening procedures in the laboratory. Expression optimization generally requires primary cultures to be grown prior to inoculation of expression culture. Both primary cultures and expression culture typically need to incubate three days each, so that one screening round will take at least one week.

In this study, we used IMAPlate 5RC96, a multifunction miniature analytical platform, ${ }^{[9,10]}$ to perform the enzymatic assay. Due to the enhanced sensitivity and small volumes of the IMAPlate, it allowed us to reduce the expression culture to 100 $\mu \mathrm{L}$ (or even less) and to inoculate them directly with a cell colony reaching high initial cell density. Thus, it reduced the cultivation time and accelerated the screening procedure. The results from the screening of a collection of $S$. cerevisiae secreting CALB derivatives demonstrated that the IMAPlate based miniature screening procedure is a sensitive, easy-to-handle, high throughput method. It can dramatically shorten the time to result and increase the lab productivity.

\section{Material and Methods}

\section{Clone Collection}

The clone collection involved in this study results from a second generation CALB mutagenesis strategy. The input gene submitted to mutagenesis consists in a synthetic gene encoding the CALB Z derivative, which diverges from wild type CALB (wtCALB, EMBL Z30645) by three residues: valine 210, valine 221 and alanine 281 were respectively replaced by isoleucine, aspartic acid and glutamic acid.

Libraries of second generation mutant genes evolving from CALB $\mathrm{Z}$ were generated by the amplification of input gene by manganese-induced Error-prone PCR following the method of Cadwell and Joyce. ${ }^{[11]}$ Amplification added a hexa histidine encoding stretch upstream of the coding frame and restriction sites at both ends, which enables cloning of PCR products in the yeast shuttle vector pEMBLyex 2 in frame with a sequence encoding Saccharomyces cerevisiae alpha factor, driving the secretion of the recombinants product. ${ }^{[12]}$

\section{Protein Expression}

S. cerevisiae HT393 clones expressing CALB wild type (wtCALB), CALB Z or a CALB $Z$ derivative were inoculated in $2 \mathrm{~mL}$ minimal medium without uracile or leucine $(2 \%$ dextrose, $0.67 \%$ yeast nitrogen base without amino acids, $20 \mathrm{mg} / \mathrm{L}$ tryptophan, adenine, L-histidine, L-methionine, and L-lysine, $30 \mathrm{mg} / \mathrm{L}$ L-leucine) and were grown $72 \mathrm{~h}$ at $30^{\circ} \mathrm{C}$. Cell density of these primary cultures was determined spectrophotometrically at $600 \mathrm{~nm}$ and cultures were diluted to a cell density of OD 0.05 with minimal medium. $100 \mu \mathrm{L}$ of the diluted primary cultures were centrifuged $5 \mathrm{~min}$ in a V-bottom microtiter plate at $4500 \mathrm{~g}$. The resulting pellet figures the number of cells of a typical $S$. cerevisiae colony on plate.

For inoculation of miniature expression cultures, we suspended the pellets in $100 \mu \mathrm{L}$ (2\% galactose, $1 \%$ yeast extract, bactopeptone $2 \%, 100 \mathrm{mM}$ sodium phosphate $\mathrm{pH}$ 6.0). Expression cultures were grown directly in V-bottom plate for 24 or $72 \mathrm{~h}$ at $30^{\circ} \mathrm{C}, 100 \mathrm{rpm}$. 
In order to compare with a standard procedure, we also grew $2 \mathrm{~mL}$ expression cultures. $100 \mu \mathrm{L}$ of primary cultures diluted to a cell density of OD 0.05 was directly given to $2 \mathrm{~mL}$ expression medium in a $15 \mathrm{~mL}$ culture tube. The $2 \mathrm{~mL}$ expression cultures were grown for $72 \mathrm{~h}$ at $30^{\circ} \mathrm{C}$, $100 \mathrm{rpm}$.

\section{Activity Assay}

For IMAPlate based activity assay, 10 $\mu \mathrm{L}$ supernatant were transferred to the upper well chamber of an IMAPlate, whose lower chamber open bottoms were provisionally sealed with paraffin film (Fig. 1). After mixing with $10 \mu \mathrm{L}$ of substrate solution (10 mM Tris-HCl pH 7.4, 1\% DMSO, $0.1 \% p$-nitrophenyl phosphate), the paraffin film was removed and the reaction solution allowed to flow into the lower chamber of the IMAPlate where the reaction took place. The increase in absorbance was measured at $405 \mathrm{~nm}$ for $10 \mathrm{~min}$.

For conventional plate based assay, 50 $\mu \mathrm{L}$ supernatant were mixed with the same amount of substrate solution in a flat bottom transparent 96-well microtiter plate and the increase in absorbance was measured at $405 \mathrm{~nm}$ for $10 \mathrm{~min}$.

Initial velocities were calculated over the first two minutes in the linear OD range. Activity yielded by supernatants of a clone secreting alpha factor peptide without CALB fusion was subtracted from these values to obtain CALB-depending activity titers.

Instrumentation included an Infinite M200 UV spectrophometric plate reader Model 160U (Tecan, Switzerland) for conventional plate assay and a PowerWave XS2 plate reader (Biotek, USA). All reagents were obtained from Sigma Chemical Company.

\section{Results}

A concern in this work is the standardization of inoculation procedure of expression cultures. S. cerevisiae colonies vary slightly in size. On minimal medium plates, single cells form colonies with diameters approaching $1 \mathrm{~mm}$ after three days' growth. Actually, in our former studies we did not notice any evidence for a correlation between the size of colony involved in the inoculation and the activity titer yielded by the miniature expression culture resulting from its inoculation. We observed that in miniature cultures, growth is strongly limited and cell density stops increasing definitively before $24 \mathrm{~h}$ are passed. This implicates that after $24 \mathrm{~h}$ the miniature cultures grown under strictly identical conditions have all reached the same limited cell density. Therefore, irreproducibility in results due to variation in size of colonies is not expected.
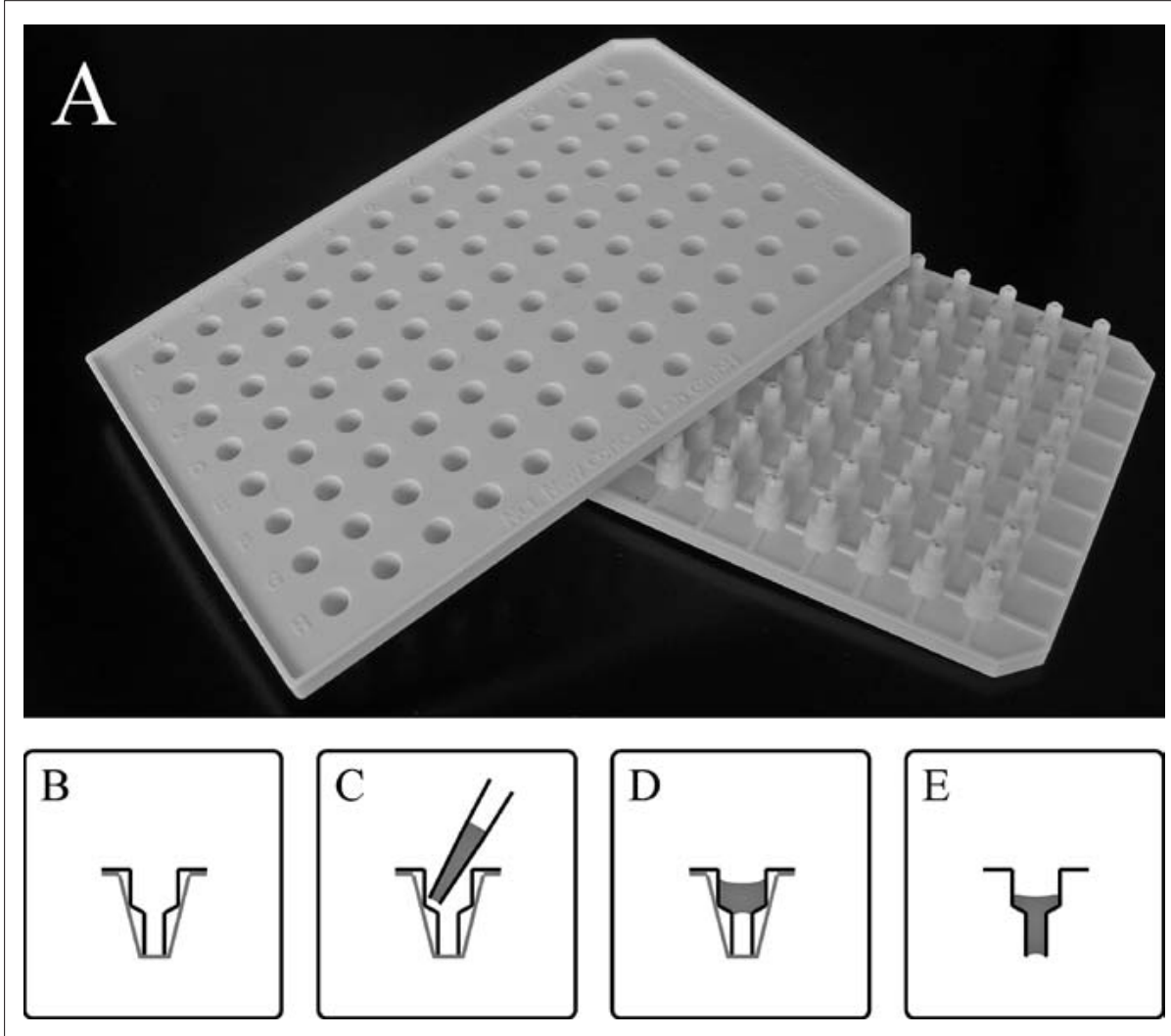

Fig. 1. IMAPlate ${ }^{\mathrm{TM}}$ 5RC96. A: The wells of this 96-well compatible plate device are opened at the bottom and consist in capillary of $5 \mathrm{~mm}$ height and $5 \mu \mathrm{L}$ capacity. B: For this application, the wel bottom is sealed with a paraffin film. C, D: Sample and substrate solution are loaded with a multi pipetting device and mixed in the upper well chamber, where reaction starts. E: Paraffin film seal is removed. Reaction solution is displaced by capillarity and fills the lower chamber. The reaction can be followed by spectrophotometer in a standard plate reader.

Nevertheless, we decided to define standard conditions to be applied to this study. We estimated the cell amount contained in a typical yeast colony by picking up 12 colonies with a diameter around 1 $\mathrm{mm}$ on our selective plates (consisting in minimal medium containing $2 \%$ agar agar) and suspending them in $100 \mu \mathrm{L}$ of minimal medium. The average optical density from these suspensions at $600 \mathrm{~nm}$ was about 0.05 . Therefore we adapted our inoculation procedure for this study to the protocol described in the material and method section, which enables us to strictly control the amount of cells given to the expression culture.

For the titration of miniature cultures, we proceeded to the controlled inoculation of four cultures of $100 \mu \mathrm{L}$ expression medium in a V-bottom microtiter plate for each clone of our collection. Activity titers were measured in IMAPlate after $24 \mathrm{~h}$ and $72 \mathrm{~h}$. The average values are represented in Fig. 2. The error bars correspond to standard deviations among the clone quadruplicates.

The gain in sensitivity of the IMAPlate based assay toward the conventional plate based assay was assessed by testing supernatants resulting from centrifugation of 2
$\mathrm{mL}$ expression cultures incubated at $30^{\circ} \mathrm{C}$, $100 \mathrm{rpm}$ for 3 days (Fig. 3). Cultures were inoculated in duplicate and supernatant were titrated in duplicate. Presented values represent the mean of the four activity titers measured for each clone and the error bars corresponds to standard deviations among them.

IMAPlate based assay yields approximately 2 to 3 -fold higher activity titers than conventional plate based assay. This increase is expected because of the longer light path length of IMAPlate. For a volume of $5 \mu \mathrm{L}$, IMAPlate can already provide an optical path length of $5 \mathrm{~mm}$. In comparison, a volume of $100 \mu \mathrm{L}$ in a conventional plate provides only an optical path length of $3 \mathrm{~mm}$.

\section{Discussion}

The assay that we present in this study is dedicated to the screening of second generation CALB mutants. The input gene submitted to mutagenesis encodes for CALB $\mathrm{Z}$, a wtCALB derivative that diverges by three residues (V210I, V221D, A281E). This mutant has been described by Zhang et al. in 2003 under the appellation 195F1. ${ }^{[6]}$ 


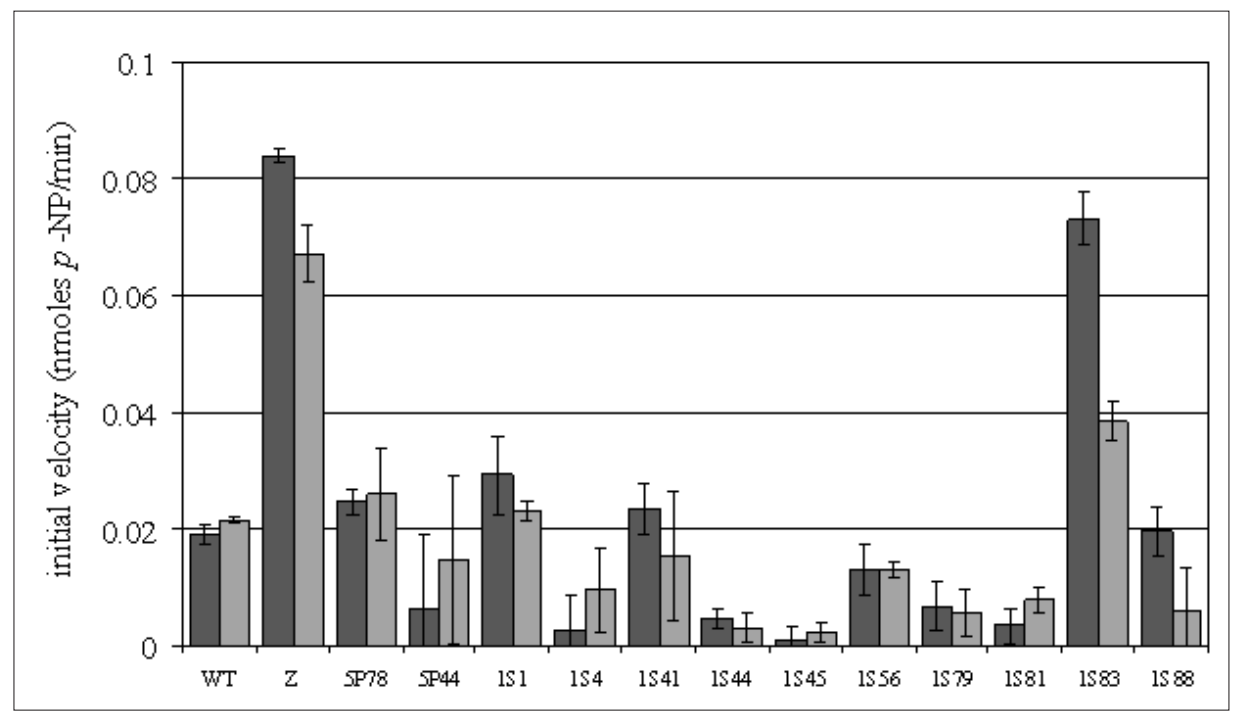

Fig. 2. Determination in IMAPlate of activity titers of miniature culture supernatants. Selected $S$. cerevisiae clones secreting CALB derivatives in medium were inoculated in $100 \mu \mathrm{L}$ expression medium and incubated at $30^{\circ} \mathrm{C}, 100 \mathrm{rpm}$ in a V-bottom microtiter plate. $10 \mu \mathrm{L}$ of culture supernatants were tested in IMAPlate after $24 \mathrm{~h}$ (dark grey bars) and $72 \mathrm{~h}$ (light grey bars) for their CALB derivative depending production of $p$-nitrophenol $(p-N P)$ in presence of $p$-nitrophenyl butyrate by following absorbance increase at $405 \mathrm{~nm}$.

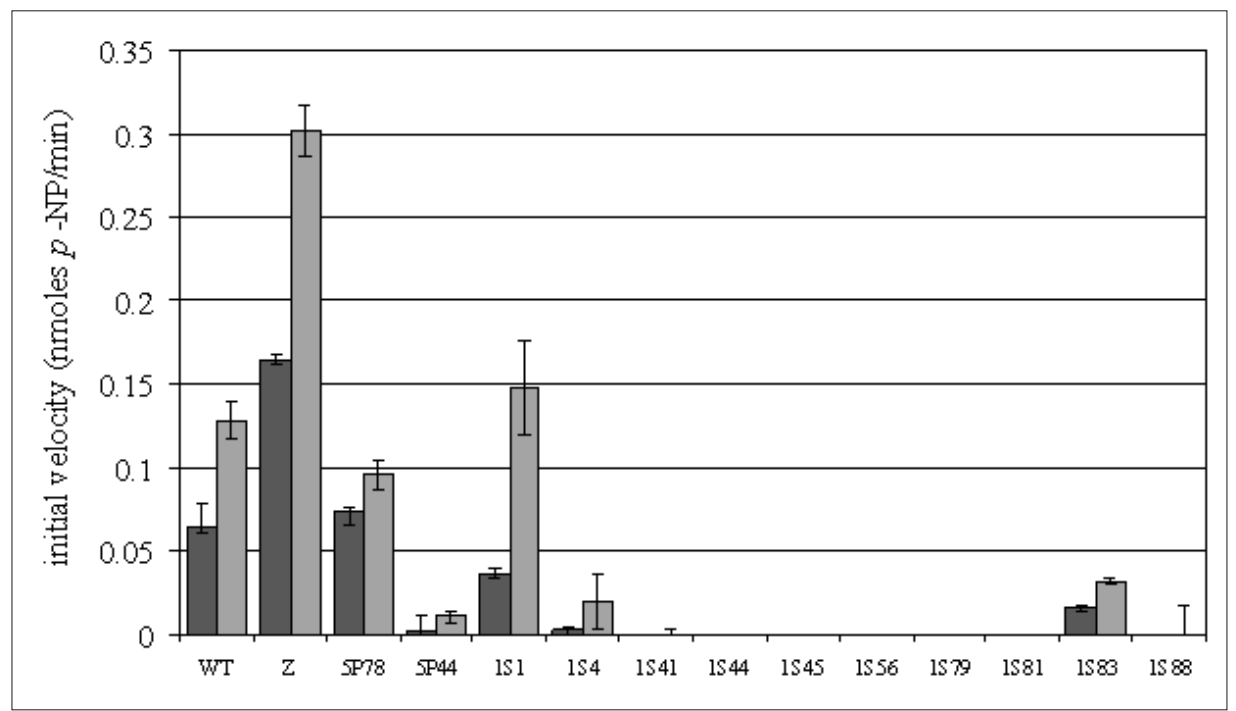

Fig. 3. Comparison between IMAPlate and conventional plate for determination of activity titers of small cultures supernatants. Colonies of selected $S$. cerevisiae clones secreting CALB derivatives in medium were inoculated in $2 \mathrm{~mL}$ expression medium and incubated at $30^{\circ} \mathrm{C}, 100 \mathrm{rpm}$ in $15 \mathrm{~mL}$ culture tubes during $72 \mathrm{~h} .50 \mu \mathrm{L}$ and $10 \mu \mathrm{L}$ of culture supernatants were tested in conventional plate (dark grey bars) and IMAPlate (light grey bars), respectively, for their CALB derivative depending production of $p$-nitrophenol $(p-N P)$ in presence of $p$-nitrophenyl butyrate by following absorbance increase at $405 \mathrm{~nm}$.

It shows increased resistance to heat inactivation and near twice the specific activity of wild type. We observed that in large cultures (more than $250 \mathrm{~mL}$ ), the increased stability of CALB Z leads to an accumulation of recombinant protein resulting after three days incubation in activity titers in supernatant that surpasses by a factor 16 at least the activity titer yielded by wtCALB expressing clones. We have observed very similar features while expressing these genes in Escherichia coli and Pichia pastoris based expression systems. Purifica- solution for the catalysis of transesterifications involving primary alcohols as cosubstrates. Therefore, the purpose of our mutagenesis strategy is to conserve the remarkable properties of CALB Z in terms of stability in water and to further develop its specific activity.

Attempts in our laboratory to combine the properties of CALB $\mathrm{Z}$ with those of other CALB derivatives in hybrid constructs combining the respective mutations systematically yielded proteins with dramatically lower performances than CALB $\mathrm{Z}$, despite of the fact that the resulting hybrids diverges from CALB $\mathrm{Z}$ only by one or two residues. Consistently with these observations, screening error-prone PCR produced CALB Z generation mutants yields barely qualitative features in terms of activity titer, consisting in seemingly unaffected CALB Z-like clones on one side and altered clones with dramatically lower or undetectable titers on the other side. Enhancing the concentration of the mutagen in error-prone $\mathrm{PCR}, \mathrm{MnCl}_{2}$ for instance, progressively displaces the balance between these two groups, yielding more low or undetectable activity titer clones. The latter represent more than $90 \%$ of the tested clone population in our experiments in the error-prone PCR conditions defined by Cadwell and Joyce. ${ }^{[11]}$ Our observations reveal a 'genetic instability' of the CALB Z properties and calls for an adaption of our screening assay to take account of this fact.

Due to the miniature feature of IMAPlate, we can use very small supernatant volumes to measure activity titers and to consequently miniaturize the upstream processing, reducing the expression cultures to $100 \mu \mathrm{L}$ or even less. These cultures can be conveniently incubated in a V-bottom 96-well microtiter plate. The procedure may be easily adapted for automated high throughput. Actually, culture volume is not a limiting factor per se, but the starting cell amount is. The advantage in miniaturizing culture volume provides a significantly high starting cell density and thus shortens the expression time. In the study, we inoculated the number of cells corresponding to an entire colony of a typical $S$. cerevisiae clone. The results obtained from the IMAPlate showed that it can clearly detect wtCALB and CALB $\mathrm{Z}$ activity in supernatant in less than $24 \mathrm{~h}$ cultivation.

Our observations suggest that the screen cycle may be completed even faster if the entire colony is directly inoculated in smaller expression medium volume, for example $25 \mu \mathrm{L}$. The reduced expression time would also be favorable to study some expressed proteins that may have negative effects on the cell viability for long incubation.

It has to be pointed out that longer incubation of miniature cultures did not yield 
higher activity titers. This is probably due to limitations by miniature expression environment and may depend on air circulation, nutrition or cell density. These results corroborate our previous assumption that growth limit is reached in less than $24 \mathrm{~h}$ in our miniature culture format. It is also remarkable that conventional based assay failed to deliver significant and consistent values of the miniature culture titers (data not shown).

Indeed, activity titers in $2 \mathrm{~mL}$ culture supernatants after 3 day incubation are significantly higher than activity titers of miniature culture supernatants after $24 \mathrm{~h}$ incubation for the most active clones, but very low activities are not detected, independent of the plate system.

In conclusion, our miniaturized IMAPlate-based method is fast, reliable, reagent saving, efficient and easy to handle. The method should be suitable for a primary screening for the CALB Z derived activity titer in less than one day directly from $S$. cerevisiae colonies. Therefore, it can markedly reduce the screening time and enhance lab productivity. Our approach should be generally applicable to other secreting enzyme screening and even for non-secreting enzyme screening with minor changes in the upstream procedure.

\section{Acknowledgements}

The authors thank Daniele Tobler and Peter Spies for expert technical assistance.

Received: September 6, 2010

[1] A. Robles-Medina, P. A. Gonzáles-Moreno, L. Esteban-Cerdán, E. Molina-Grima, Biotechnol. Adv. 2009, 27, 398.

[2] A. R. Macrae, A. R. Hammond, Biotech. Genet. Eng. Rev. 1985, 3, 193.

[3] K. E. Jaeger, T. Eggert, Curr. Opin. Biotechnol. 2002, 13, 390.

[4] Z. Qian, C. J. Fields, S. Lutz, ChemBioChem 2007, 8, 1989

[5] A. O. Magnusson, J. C. Rotticci-Mulder, A. Sanragostino, K. Hult, ChemBioChem 2005, 6, 1051.

[6] N. Zhang, W. C. Suen, W. Windsor, L. Xiao, V. Madison, A. Zaks, Protein Eng. 2003, 16, 599.

[7] S. Tamalampudi, M. M. Talukder, S. Hama, T. Tanino, Y. Suzuki, A. Kondo, H. Fukuda, Appl. Microbiol. Biotechnol. 2007, 75, 387.

[8] T. Tan, M. Zhang, B. Wang, C. Ying, L. Deng, Process Biochem. 2003, 39, 459.

[9] P. Spies, G. J. Chen, D. Gygax, Anal. Biochem. 2008, 382, 35.

[10] P. Spies, R. Müller, G. J. Chen, D. Gygax, Anal. Biochem. 2010, 397, 48.

[11] R. C. Cadwell, G. F. Joyce, Genome Res. 1994, 3, 136.

[12] G. Lipps, R. Füllkrug, E. Beck, J. Biol. Chem. 1996, $271,1717$. 